\title{
Urgences
}

\section{Au rythme d'aujourd'hui (extrait)}

\section{Jean-Yves Dupuis}

Numéro 11, 3e trimestre 1984

URI : https://id.erudit.org/iderudit/025171ar

DOI : https://doi.org/10.7202/025171ar

Aller au sommaire du numéro

Éditeur(s)

Urgences

ISSN

0226-9554 (imprimé)

1927-3924 (numérique)

Découvrir la revue

Citer ce document

Dupuis, J.-Y. (1984). Au rythme d'aujourd'hui (extrait). Urgences, (11), 33-46.

https://doi.org/10.7202/025171ar

Ce document est protégé par la loi sur le droit d'auteur. L'utilisation des services d'Érudit (y compris la reproduction) est assujettie à sa politique d'utilisation que vous pouvez consulter en ligne.

https://apropos.erudit.org/fr/usagers/politique-dutilisation/
Cet article est diffusé et préservé par Érudit.

Érudit est un consortium interuniversitaire sans but lucratif composé de l’Université de Montréal, l'Université Laval et l'Université du Québec à Montréal. Il a pour mission la promotion et la valorisation de la recherche. https://www.erudit.org/fr/ 
JEAN-YVES DUPUIS

Au rythme d'aujourd'hui (Extrait) 
Tôt un samedi, la sonnerie de la porte a résonné dans la chambre. J'y vais, a dit Caroline. Elle s'est levée péniblement et a enfilé sa jaquette de flanelle à petits pois qui ressemblait à un torchon tellement elle était vieille et usée. Ce matin il y avait même l'un de ses mamelons qui ressortait par un trou. Je le lui ai dit. Elle I'a caché en souriant. Elle est revenue un moment sur le lit et m'a embrassé sur la bouche. Ohhh! elle était tellement chaude. Enfin! Et puis elle a dit: Qui ce peut être à cette heure-ci?

Comme je n'ai pu réussir à refermer l'oeil, je me suis levé et suis allé à la cuisine pour prendre un café noir. France, la plus jeune soeur de Caroline, était débarquée chez nous. Tout de suite je me suis fait apostropher par Caroline:

\section{- Tu ne sais pas ce que ma petite soeur a fait?}

Calculant l'indignation de Caroline, ce devait être sûrement abominable. Je n'avais pas très envie de savoir mais Caroline ne me demandait pas mon avis. Voilà I'histoire: La petite soeur avait été surprise par papa Lapierre en train de regarder des films pornos à la télévision. Il lui avait foutu une râclée et elle rappliquait chez nous pour fuir les fureurs de son petit papa adoré.

- Elle n'a que treize ans! Tu imagines?... Regarder des films pornos! Peuh! Moi, à ton âge, tout ce que j'avais pour faire mon éducation sexuelle, c'était le petit dictionnaire Larousse. Maudite jeunesse! Où est-ce que I'on s'en va?...

- Tu ne trouves pas que tu en fais un peu trop? a dit France calmement. Ce n'est pas la fin du monde.

Pour avoir la paix, je suis allé boire mon café sur le siège des toilettes.

Quand j'en suis ressorti, Caroline criait encore:

- En tout cas, si tu penses que je vais te garder ici!... Je ne tiens pas une garderie. Treize ans!... Ça n'a pas de sacré bon 
sens. (Etc, etc, etc,). Je vais téléphoner à la maison pour avertir que tu es ici.

Elle a téléphoné. Elle a discuté un bon moment et quand elle a eu raccroché, elle paraissait un peu plus calme. J'ai pu lui demander comment la mère avait pris l'affaire.

- Elle n'était pas très contente. Les petites fugues, ce n'était pas la première fois. Dans le temps, Sylvie aussi avait fait une escapade. Je pense qu'elle avait quinze ans. Elle s'était rendue jusqu'en Colombie-Britannique avec un garçon, pour faire la cueillette des cerises. Je me demande pourquoi elle n'avait averti personne. Mes parents l'avaient fait rechercher. Ça a été toute une histoire. Finalement Sylvie a appelé de Keremeos, elle n'avait plus un sou et voulait rentrer.

Elle a regardé France, puis lui a dit sur un ton qui n'accepte pas de réplique:

- Toi, prépare-toi! Je te ramène à North Hatley.

Caroline allait s'habiller à la chambre.

France s'est mise à pleurnicher doucement dans ses mains. Je suis venu près d'elle et je lui ai demandé:

- Tu veux que je te fasse un café?

Elle a fait signe que oui.

- Tu sais, Caroline crie un peu fort mais elle t'aime bien, j'en suis sûr.

- Écoute, demande-lui que je reste ici toute la fin de semaine. Demain... demain, je m'en irai. Je n'ai pas envie de voir mon père tout de suite. II n'y a rien à faire avec lui il ne peut jamais comprendre ce qu'on lui dit.

Bon. Caroline est revenue à la cuisine. Je lui ai fait le 
message. Elle a encore grimpé sur ses grands chevaux.

- Quoi, tu prends sa défense maintenant?

- Tout de même! j'ai dit. Elle n'a tué personne.

- Non, mais elle regarde des films pornos.

- Toi, tu ne regardes peut-être pas des films pornos mais tu fais pire encore. Est-ce que tu voudrais que je raconte à France ce que tu as fait la nuit dernière? Hein?

Caroline a esquissé un sourire.

- Bon, tu peux rester. Mais jusqu'à demain seulement. Moi je ne peux jamais dire non à personne. Je suis trop bonne. Hein, dis-le que je suis bonne!... Dis-le!

- Tu es bonne, a fait France.

— Viens m'aider. Nous allons faire une petite épicerie.

- Non, a dit France.

- Pourquoi?

- Parce que tu cries trop fort.

- Moi je crie trop fort? a dit Caroline. Allez, habille-toi, et viens avec moi. Je suis pressée.

- Non!!! ...

- Laisse-la, j'ai dit. Si elle ne veut pas y aller!... nant?

- Vous avez décidé de vous liguer contre moi mainte-

Ça ne lui arrivait pas trop souvent de prendre le mors aux dents mais alors, Caroline, elle devenait d'un pénible. 
Elle est finalement partie en claquant un peu la porte.

Je me suis assis à la table et j'ai bu mon café. France était un peu gênée. Elle a fini par demander:

- Qu'est-ce que tu penses de moi?

- Tu es une jolie fille.

Elle a souri.

- Non. Je veux dire: à cause des films pornos.

- J'm'en fous, moi. Tu peux bien regarder tous les films pornos que tu veux.

Elle a ajouté comme pour s'excuser:

- Il faut bien que je me renseigne quelque part. Personne ne veut répondre à mes questions à moi.

- Qu'est-ce que tu veux savoir?

Je la gênais, c'était évident. Elle a bégayé quelques mots qui ne voulaient rien dire. Quand elle parlait, elle plaçait sa main sur la bouche pour me cacher ses broches.

- Si tu penses que je vais demander ça à toi?

- Pourquoi?

- T'es un gars.

- Oui, je sais.

- À la télé, ils ne nous montrent jamais rien, a continué France.

- C'est un pénis que tu veux voir? 
Elle a fait signe que oui. Elle a même dit qu'à part les photos, elle n'en avait jamais vu de toute son existence.

- Tout le reste, je le sais. Ce qu'on nous montre à la télévision, c'est des filles nues. C'est bon pour les vieux bonshommes. Michelle, elle, elle a déjà couché avec un gars et elle a dit que la première fois ça lui avait fait un peu mal. J'ai hâte d'avoir son âge. Dis, est-ce que tu voudrais me le montrer, le tien?

- Quoi, mon pénis?

- Oui. Je n'y toucherai pas. Juste pour voir comment c'est fait. Je pense que je suis assez vieille pour connaître ça.

Cette fois, c'est elle qui me gênait. Mais j'ai quand même baissé mon pantalon rapidement. Que ne ferais-je pas pour l'éducation de notre belle jeunesse? France $m$ 'a examiné le machin avec curiosité.

- Je pensais que c'était plus long que ça. Oui, ceux que j'ai vu sur les photos étaient bien plus longs.

Je me suis réenculotté, un peu piqué.

- II ne faut pas que tu te fies aux revues pornographiques. Ceux qui posent là-dedans, ce sont de vrais monstres.

- En tout cas, je ne trouve pas ça très joli. Je ne sais pas pourquoi les gars en font un tel plat.

- Attends d'être plus vieille. Tu vas adorer ça.

- Ouais? Mais j'ai besoin de changer. Euh... j'ai un peu faim. Je n'ai pas déjeuner ce matin.

- Sers-toi, chère enfant.

- À la maison, c'est toujours ma mère qui me fait mes repas. Alors!... Je ne suis même pas capable de casser un oeuf. 
Finalement, je lui ai servi un bon déjeuner. Ensuite nous sommes sortis tous les deux, pour nous balader. Nous avons marché longtemps. Passant devant un marché de fruits et légumes, j'ai acheté des raisins et des bleuets. France en a tellement mangé qu'elle en a eu mal au ventre. Ça lui apprendra! Mais ça ne l'a pas empêchée de vouloir bouffer les fruits que j'avais achetés pour moi, avec l'intention de les manger plus tard. Nous avons décidé de nous arrêter à un bar, rue Sainte-Catherine, avant de revenir à l'appartement. France avait toute la bouche et le menton salis de bleuets. En nous dirigeant à une table au fond, nous sommes passés devant des gens, deux types et leurs attitrées, et ils ont ri en nous voyant. Quand nous avons été assis, France a demandé:

- C'est de toi ou de moi qu'ils ont ri?

- C'était sûrement de toi, j'ai dit. laid.

- Peuh! Pourquoi ils auraient ri de moi? Tu es bien plus

- Tu ne t'es pas regardée. T'as la gueule toute salie et ton t-shirt est dégueulasse.

La serveuse est venue prendre notre commande et quand elle a vu la tête de France, elle a pouffé de rire. Mais elle a détalé tout de suite. J'ai dit:

- Bon, qu'est-ce que je te disais?

J'ai pris un mouchoir de papier et je lui ai frotté le museau. France a dit:

- Aussi bien qu'ils rient, je leur donne des occasions.

Je n'étais pas tellement sûr qu'elle croyait ce qu'elle disait mais enfin! Elle n'avait pas l'air de vouloir en faire toute une histoire. 
Après un moment, elle a dit:

- Toi, tu es gentil. II n'y a personne qui est gentil comme toi avec moi. Tout le monde rit de moi parce que j'ai des broches. sale.

Et elle a commençé à pleurnicher dans son vieux t-shirt

- Écoute! C'est joli, des broches.

Comme connerie, c'était difficile de mieux dire. Mais France a quand même cru que je voulais lui faire un compliment.

- Tu dis ça pour me faire plaisir.

- Non, je t'assure.

- Et puis, dans la famille, personne ne s'occupe de moi. Ce que je dis n'a jamais d'importance. Tu as vu, avec Caroline, elle me prend pour un bébé.

- Euh... tu n'est pas très vieille aussi.

- J'ai treize ans!...

- Oh, excuse-moi. Cesse de pleurer.

Elle s'est essuyé le nez avec son gilet.

- J'ai des boutons.

- Où ça?

Elle m'a montré un petit point rouge près de son nez.

- Où?

- Là. 
- Je ne vois rien.

- Alors c'est que t'es aveugle!...

- Est-ce qu'il y a autre chose encore?

- Je n'aime pas mon manteau. Maman ne veut pas m'en acheter un autre. Elle dit que celui-là est encore bon.

- Qu'est-ce qu'il a?

- C'était celui de Michelle.

- Autre chose?

- Je n'ai jamais d'argent pour m'acheter des cigarettes. Personne ne veut $\mathrm{m}^{\prime}$ en donner.

- Tu fumes, toi? À ton âge!

- J'ai treize ans!...

- Oh!... J'avais oublié. Attends.

Je me suis levé pour aller chercher des cigarettes et quand je suis revenu, je lui ai mis le paquet devant elle. J'ai dit:

- Tiens!...

Elle a paru tellement contente.

- C'est vrai? Tu me donnes tout le paquet?...

- Ouais. Bon, tu étais en train d'énumérer tous tes malheurs. Où nous en étions?

- Je t'embète, hein?

- Bien non. Alors, qu'est-ce qu'il y a encore? 
- Je ne sais pas si je vais te le dire.

- Quoi?

- Je n'ai pas encore... bien... euh... tu sais... les menstruations. Toutes les autres filles les ont eues. Moi je ne fais jamais rien comme les autres. II n'y a pas assez que j'ai des broches, en plus je ne suis pas menstruée.

- Ça va venir, j'ai dit.

- Je les veux tout de suite.

- Tu devrais être contente de ne pas avoir ce genre de problèmes. Caroline, elle, n'arrête pas de sacrer contre ses "maudites menstruations", comme elle dit. Et elle a toujours un peu mal à la tête quand ces moments-là.

- Ça ne me fait rien d'avoir mal à la tête, en autant que je serai comme toutes les autres filles.

Et encore des larmes de crocodile.

$J$ avais un peu hâte de déguerpir de là. Alors j'ai demandé de partir. France a dit:

- Je ne sais pas si Caroline est revenue. Elle n'était pas de bonne humeur. Je crois que je l'ai fait fâcher.

— Ne t'en fais pas. Ça va lui passer.

- Caroline, elle se prend toujours pour ma mère. Comme si je n'en avais pas assez d'une!... II faut toujours qu'elle me fasse la morale. Ce n'est pas drôle d'avoir beaucoup de soeurs plus vieilles. Combien tu en as de soeurs, toi?

- Trois.

- Tu es chanceux. Moi j'en ai huit. Huit soeurs. Et même pas un petit frère. Ma mère aurait pu se forcer un peu, et 
ne pas faire seulement que des filles. II paraît qu'il y a une chance sur plusieurs millions, pour une femme, d'avoir neuf filles d'affilée. Et il fallait que cela tombe sur notre famille.

- J'en ai rencontré plusieurs, de tes soeurs. Et elles me paraissent formidables.

- Alors c'est que tu n'a jamais rencontré Sylvie. Elle est complètement folle, celle-là. Tu ne sais pas ce qu'elle fait?

- Non.

- Quand elle vient à la maison, et que mes parents ne sont pas là, elle se baigne toute nue dans la piscine. Et puis elle couche avec des femmes. Elle me l'a dit. Pourquoi tu ris?

- Pour rien. Qu'est-ce qu'elle fait encore, Sylvie?

- Elle parle tout le temps. Et elle n'arrête pas de se moquer de moi. Un bon jour, je vais lui donner un coup de poing sur la gueule. Oui! C'est ce qu'elle mérite.

- Pourquoi elle rit de toi?

- Pour toutes sortes de raisons. Oui, un jour je vais lui donner un coup de poing sur la gueule. mander:

Elle s'est tue un moment. J'en ai profité pour lui de-

- À quoi tu pensais quand tu es arrivée chez nous?... Que Caroline allait t'héberger à I'avenir?

- Bien... euh... Mon père $m$ 'a donné une fessée. Peuh!... Je n'ai pas réfléchi. Il était une heure de la nuit. Je suis partie de la maison en courant, je ne sais pas s'il m'a suivie, je me suis cachée dans le cimetière jusqu'au matin, puis j'ai pris I'autobus.

- Tu n'as pas eu peur? 
- Pourquoi j'aurais eu peur? Souvent, avec mes amis, nous jouons à la cachette dans le cimetière. Le curé, une fois, s'est mis à courir après nous pour nous dire de sortir de là. C'était drôle! II ne courait pas vite, il est trop gros, il souffle fort, j'ai toujours peur qu'il crève. Ma bière est déjà vide. J'ai bu trop vite. Ça ne m'arrive pas souvent de boire de la bière.

- Nous y allons?...

Tous les deux, nous sommes revenus à l'appartement en début d'après-midi. Caroline a tout de suite commençé à m'engueuler parce que j'avais soi-disant "entraîné" France et que maintenant elle était pompette. C'est vrai que la petite soeur était juste un peu saoule mais il n'y avait pas besoin de faire toute une histoire. C'est ce que je lui ai dit. Alors elle a crié:

- Tu crois ça, hein? Tu lui achètes des cigarettes, tu la fais boire... Tu as une drôle de conception de la façon d'éduquer des enfants.

- Je ne suis pas une enfant, a dit France, péniblement.

- Oh toi, tu ferais aussi bien d'aller te coucher. Avec la gueule que tu as!... Si maman était ici, tu...

- Je voudrais bien voir que tu ailles raconter ce que j'ai fait parce que... parce que, moi aussi, je pourrais avoir beaucoup à dire.

- Quoi, par exemple?

- Peuh!...

Caroline a parlé plus doucement:

- De toute façon, couche-toi pendant quelques heures, jusqu'au souper, et je ferai quelque chose de spécial.

- Bon!... 
France a trotté jusqu'à la chambre de Caroline. Presqu'immédiatement, elle est revenue en disant:

- Je ne peux pas dormir dans ton lit. Les draps sont sales, il y a plein de taches de sang et... des taches blanches...

Caroline a souri: Prends-en des propres dans l'armoire!...

Puis elle a ajouté, pour moi:

- Dans la famille, il y a toujours l'une ou l'autre qui a des petits problèmes, et je ne sais pas pourquoi, c'est toujours moi qui écope de leurs confidences. Je dois avoir l'air d'une bonne mère de famille. Avec mes gros seins aussi!...

Jusqu'au souper, Caroline n'a plus trouvé rien à faire. Elle s'est installée devant la télévision, et même si on ne présentait que du sport, elle n'a pas voulu en bouger. Elle a regardé la partie de baseball jusqu'à la dernière minute. Et puis elle est sortie quelques instants. Elle est revenue avec de la bière, un roman policier, et un gros sac de chips. Elle s'est réinstallée sur le divan pour lire, en disant:

- Ne me dérange pas. Je lis.

Elle m'énervait...

J'ai choisi d'aller faire une petite sieste moi aussi. 


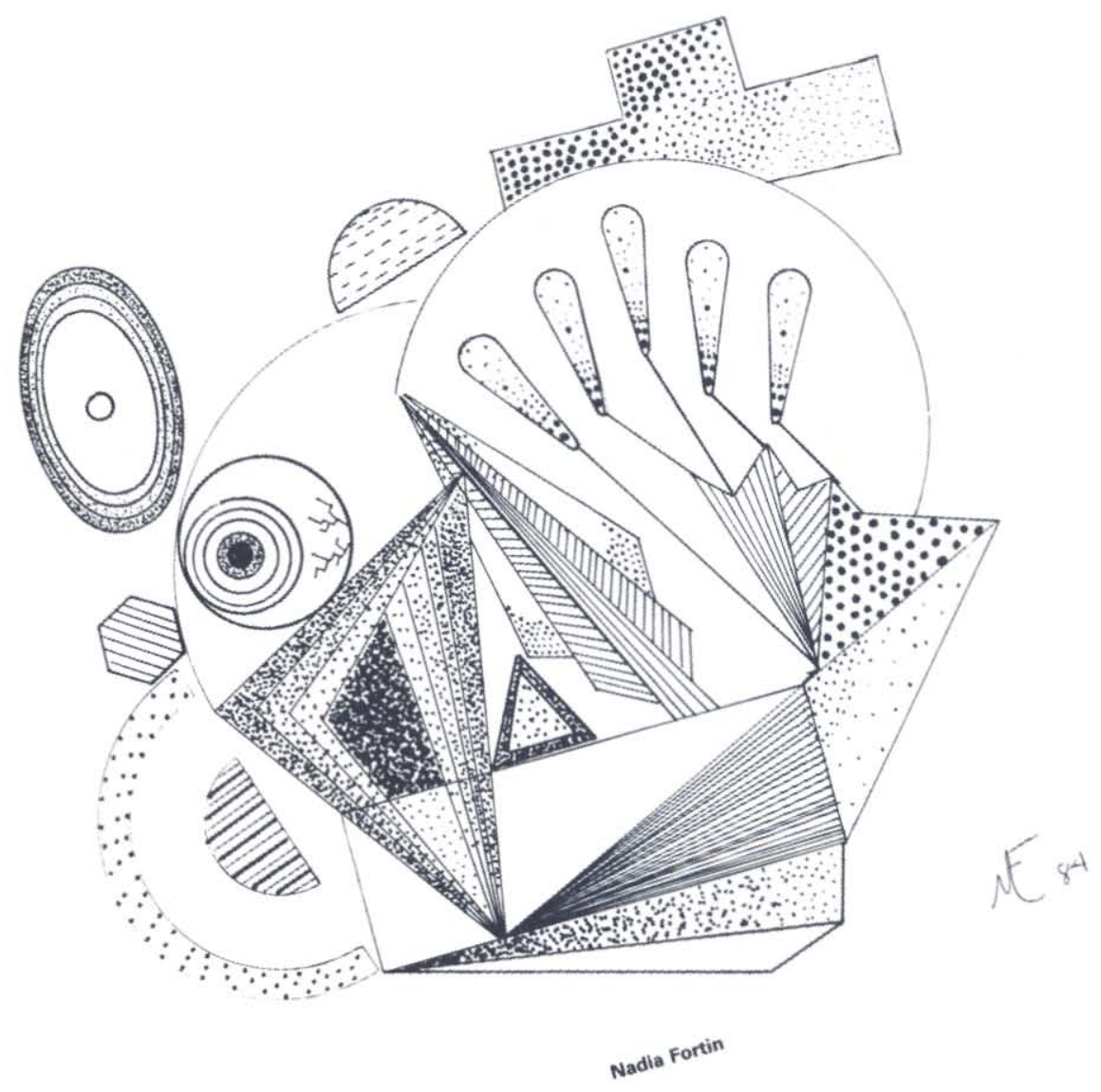

